\title{
La greffe de moelle osseuse dans l'adrénoleucodystrophie
}

$\mathbf{L}$ a première tentative d'effectuer une greffe allogénique de moelle osseuse dans une maladie dégénérative héréditaire du système nerveux central $(\mathrm{SNC})$ remonte à 1980. Elle fut réalisée au Westminster Children's Hospital chez un enfant atteint de maladie de Hurler, une maladie lysosomale qui débute vers l'âge de 1 an et entraîne le décès avant 10 ans par surcharge de mucopolysaccharides dans le SNC, l'os, le foie, la rate et la cornée. L'enfant fut greffé à l'âge de 9 mois avec la moelle osseuse de sa mère (hétérozygote) à un stade où il ne présentait encore que peu de signes cliniques. Agé actuellement de 20 ans, ce patient a une intelligence dans les limites de la normale, lui permettant d'avoir une vie active pratiquement normale malgré la persistance de complications osseuses (mal corrigées par la greffe de moelle osseuse, on le sait aujourd'hui avec le recul chez d'autres patients greffés atteints de cette maladie). A cette époque, on n'avait pas encore établi avec certitude que des cellules de la lignée monocyte-macrophage (on ne sait toujours pas aujourd'hui à quel stade exact de différenciation) pouvaient normalement traverser la barrière hémato-encéphalique sans que celleci soit altérée, et se différencier in situ en macrophages cérébraux ou microglie [1, 2]. L'idée était pourtant lancée que ce mode de thérapie pouvait permettre d'apporter dans le SNC, le foie, la rate et, bien sûr, la moelle osseuse, l'enzyme normale $(\alpha$-L-iduronidase) dont l'activité est déficitaire dans cette maladie. Dans la mesure où l'on savait que les enzymes lysosomales sont des protéines solubles qui peuvent être sécrétées et recaptées par voie endocytique par d'autres cellules, on avait l'espoir que ce type de greffe pourrait permettre d'apporter dans les neurones du SNC une certaine quantité d' $\alpha$-L-iduronidase normale et empêcher ainsi leur dégénérescence. La première tentative de greffe de moelle osseuse dans l'adrénoleucodystrophie (ALD) fut faite en 1983 [3]. A cette époque, on savait seulement que cette maladie dégénérative de la myéline du SNC se caractérisait par une accumulation modérée (X3 à 5) d'acides gras à très longue chaîne (AGTLC) dans tous les tissus, y compris le cerveau, mais on n'avait aucune information sur la nature de la protéine codée par le gène de l'ALD. Le pari thérapeutique soustendant cette première tentative de greffe était le même que dans la maladie de Hurler : en renouvelant par la greffe de moelle osseuse le pool de macrophages cérébraux, on espérait apporter dans le SNC une certaine quantité "d'enzyme" normale et ainsi y abaisser voire normaliser les concentrations d'AGTLC qui pouvaient avoir un rôle "toxique" sur les membranes myéliniques. Cette première greffe fut réalisée chez un garçon présentant une atteinte démyélinisante pariéto-occipitale déjà évoluée. Elle permit d'abaisser considérablement les taux plasmatiques d'AGTLC chez ce patient (par apport de macrophages normaux dans le foie). Elle fut cependant un échec sur le plan clinique: le patient décéda quelques mois plus tard de sa maladie neurologique et on se demanda même si la greffe n'avait pas accéléré le processus de démyélinisation cérébrale. En 1987, lors d'une réunion d'hématologistes spécialisés dans la greffe de moelle osseuse au Johns Hopkins Hospital, le sentiment des participants était qu'il serait inutile de répé- ter cette triste expérience, vu l'inefficacité apparente de la greffe de moelle osseuse dans l'ALD.

Une nouvelle greffe de moelle osseuse fut cependant réalisée à Paris en avril 1988 chez un patient ALD présentant une atteinte cérébrale débutante. Une complète disparition des lésions de démyélinisation fut observée 18 mois après la greffe, associée à une amélioration significative des capacités cognitives du patient [4]. Douze ans plus tard, ce patient, maintenant adulte, reste totalement asymptomatique sur le plan neurologique et a une vie strictement normale. Plus de 100 patients ALD ont été greffés depuis, et des résultats récemment publiés (voir ci-dessous) confirment l'efficacité à long terme de la greffe allogénique de moelle osseuse dans l'ALD [5]. Les connaissances sur l'ALD ont également sensiblement progressé depuis 1983, et il est probable que les mécanismes sous-tendant l'efficacité de la greffe de moelle osseuse dans cette maladie soient totalement différents de ceux qui peuvent expliquer son efficacité dans les maladies lysosomales avec atteinte cérébrale.

\section{Que sait-on de l'ALD aujourd'hui ?}

De transmission récessive liée à l'X, l'ALD se caractérise par une grande variation phénotypique $[6,7]$ avec deux formes cliniques principales: une forme cérébrale qui atteint surtout des garçons entre 5 et 12 ans (40\% des cas), conduisant au décès en 2 à 5 ans, et une forme adulte, appelée adrénomyéloneuropathie (AMN, 60\% des cas), qui se traduit par la survenue d'une myélopathie plus ou moins sévère entre 20 et 40 ans. L'ALD se caractérise sur le plan biochimique par une accumula- 
tion d'AGTLC dans tous les tissus et le plasma, qui est secondaire à un déficit de leur $\beta$-oxydation dans les péroxysomes. Il n'existe aucune corrélation entre le phénotype clinique, le phénotype biochimique et le type de mutation du gène ALD [8], et l'on a invoqué l'existence de gènes "modificateurs" et/ou de facteurs de l'environnement. Le phénotype de base de l'ALD est probablement représenté par l'atteinte de la moelle épinière (conduisant à l'apparition d'une paraplégie spastique) avec une pénétrance complète après l'âge de 40 ans. Il en est de même chez la souris ALD [9-11]. L'atteinte cérébrale démyélinisante de l'ALD est probablement un phénomène secondaire largement sous l'influence de facteurs environnementaux avec deux pics d'âge de survenue: entre 4 et 10 ans (formes cérébrales de l'enfant), mais aussi à l'âge adulte, entre 30 et 40 ans, puisque $35 \%$ des patients atteints d'AMN développent secondairement une atteinte cérébrale démyélinisante qui, à long terme, a le même pronostic que chez l'enfant.

Le gène ALD code pour un hémitransporteur ABC (ATP-binding-cassette), localisé dans la membrane des péroxysomes [12] et qui forme des homodimères et des hétérodimères avec deux autres transporteurs ABC péroxysomaux apparentés (ALD related et PMP70) [13]. La fonction exacte de l'ALDP n'est toujours pas connue. Il existe dans l'ALD un déficit d'activation des AGTLC en dérivés CoA (première étape de leur dégradation par $\beta$-oxydation dans les péroxysomes), expliquant leur accumulation dans les tissus. Il est possible que l'ALDP interagisse avec l'enzyme responsable de cette activation (AGTLC-CoA synthétase), mais l'ALDP n'a aucun rôle dans son ciblage dans les péroxysomes. L'idée prévalente actuelle est que l'ALDP importerait les AGTLC dans les péroxysomes pour qu'ils y soient transformés en dérivés CoA. Il est possible aussi que l'ALDP importe un facteur nécessaire à l'activité normale de l'AGTLC-CoA synthétase. De manière plus importante encore, le fait que l'ALDP forme différentes combinaisons de dimères soulève la sieurs fonctions (transport d'acides gras ou de substrats de nature différente des AGTLC).

Chez l'homme comme chez la souris, l'ALDP n'est pas exprimée dans les neurones mais uniquement dans les cellules gliales et l'endothélium du SNC [14]. L'ALDP est fortement exprimée dans les astrocytes et la microglie mais, de manière surprenante, faiblement et uniquement dans une sous-population d'oligodendrocytes (voies pyramidales, corps calleux, commissure antérieure). Bien que l'on observe une disparition des oligodendrocytes par apoptose [15], il n'est pas du tout certain que l'atteinte de ces cellules soit primitivement la conséquence de l'absence de protéine ALD fonctionnelle. Les mécanismes responsables de la démyélinisation cérébrale de l'ALD restent par ailleurs toujours inconnus. On invoque souvent l'accumulation intra-cérébrale des AGTLC qui pourrait avoir un effet déstabilisant sur les membranes myéliniques [16]. La souris ALD ainsi que d'autres souris invalidées pour d'autres gènes impliqués dans la $\beta$ oxydation des AGTLC (acyl-CoA oxidase, protéine bifonctionnelle) ne développent cependant aucun signe de démyélinisation cérébrale. Le seul modèle suggérant un rôle "toxique" des AGTLC est un mutant ("bubblegum») de la drosophile qui présente un déficit d'une AGTLC-CoA synthétase (différente de celle de l'homme et de la souris), une accumulation d'AGTLC et une dégénérescence post-natale précoce des photorécepteurs de la rétine [17]. De manière importante cependant, le traitement pré-natal des larves «bubblegum » mutantes avec de l'acide oléique permet de prévenir l'accumulation d'AGTLC et la pathologie rétinienne. Il est cependant plutôt probable que chez l'homme (comme chez la souris), l'accumulation d'AGTLC n'ait, en soi, que peu d'effets sur la myéline du SNC, du moins initialement. On pourrait en revanche envisager que, chez les patients ALD, les processus de réparation de la myéline du SNC soient inefficaces en cas d'agression même minime par un virus ou un toxique de l'environnement $[8,18]$.
L'absence de protéine ALD fonctionnelle dans les cellules gliales, et plus particulièrement les macrophages cérébraux (microglie), pourrait non seulement perturber ce processus de réparation mais aussi pérenniser et aggraver les lésions initiales de démyélinisation qui surviennent suite à une "agression" externe. En faveur d'un dysfonctionnement primitif de la microglie est l'observation d'une anomalie de la régulation de production du TNF- $\alpha$, dans les monocytes de patients ALD et ce quel que soit le phénotype clinique [19].

L'efficacité de la greffe de moelle osseuse dans l'ALD (voir ci-dessous) est aussi en accord avec cette hypothèse. Celle-ci est d'autant plus surprenante que l'on sait aujourd'hui que la protéine ALD est une protéine non sécrétée (contrairement aux enzymes lysosomales) et qu'il n'existe aucune coopération intercellulaire dans le métabolisme des AGTLC (en d'autres termes, les oligodendrocytes, les astrocytes, les macrophages cérébraux et les cellules endothéliales ne sont capables de dégrader que leur propre AGTLC et pas ceux des autres cellules). Il est donc peu probable que l'efficacité de la greffe de moelle osseuse dans l'ALD soit simplement due au "remplacement» de macrophages mutés par des macrophages normaux du donneur capables de dégrader normalement l'excès d'AGTLC présent dans le cerveau des patients. Des expériences en cours visent ainsi à prouver que la greffe de moelle osseuse dans l'ALD représente une véritable forme de thérapie cellulaire qui permet de remplacer progressivement les cellules (macrophages/microglie) dont le dysfonctionnement est responsable d'un déficit de réparation de la myéline en cas d'agression exogène.

\section{La greffe de moelle osseuse} dans l'ALD : état actuel et perspectives

Après le premier résultat encourageant obtenu en 1990 [4], des résultats récents ont confirmé l'efficacité à long terme de la greffe de moelle osseuse dans l'ALD [5]. Dans une série de 18 patients ALD greffés entre 1988 et 1992, six sont décédés : 2 de complications de greffe (mala- 
die veino-occlusive, maladie du greffon contre l'hôte) et 4 de leur maladie neurologique. Ces 4 patients présentaient tous un stade évolué de leur maladie au moment de la greffe. Parmi les 12 patients ayant survécu à la greffe et avec un recul de 5 à 10 ans (en fait 7 à 12 actuellement), l'IRM cérébrale a montré une disparition des lésions de démyélinisation chez 2 patients, une amélioration chez un patient et une stabilisation de ces lésions chez les 9 autres, après en général une période initiale d'aggravation modérée durant les 1218 mois suivant la greffe. Les fonctions motrices sont restées normales ou ont été améliorées chez tous les patients greffés. Une aggravation des fonctions cognitives a été observée initialement chez 5 patients, suivie d'une stabilisation. Chez les 7 autres, elles se sont soit améliorées, soit stabilisées. Les effets bénéfiques de la greffe ont été confirmés sur une plus large série de patients $(n=126)$ mais avec un moindre recul [20].

L'expérience acquise démontre que la greffe n'est cependant efficace qu'à un stade précoce de la maladie, c'est-à-dire celui où les lésions de démyélinisation progressent lentement et restent peu étendues, entraînant essentiellement des déficits cognitifs modérés sans troubles neuro-sensoriels (hémiplégie, ataxie, atteinte visuelle et auditive centrale). A ce stade, les lésions de démyélinisation ne s'accompagnent pas encore de réaction inflammatoire. L'apparition d'une réaction inflammatoire traduit un tournant décisif dans l'évolutivité de la maladie car elle s'accompagne toujours d'une dégradation massive des fonctions cognitives et motrices conduisant au décès en quelques mois ou années. Bien que n'ayant toujours pas de marqueurs biologiques de l'évolutivité de la maladie, la progression et la sévérité des lésions de démyélinisation peuvent être prédites à court-moyen terme de manière individuelle à partir de critères cliniques et neuroradiologiques. Cette évaluation du stade évolutif de la maladie est absolument essentielle pour poser l'indication d'une greffe de moelle osseuse. Comme indiqué précédemment, celle-ci est inefficace à un stade évolué de la maladie et cette thérapeutique reste toujours associée à un risque lourd de mortalité (15 à $30 \%$ chez l'enfant, suivant que le donneur est apparenté ou non). Il faut donc anticiper ce que sera l'évolution spontanée de la maladie pendant les 12-18 mois qui suivront la greffe pour espérer obtenir un bénéfice clinique. L'expérience indique en effet que la majorité des patients ALD greffés continuent de s'aggraver en période post-greffe immédiate. Bien que l'on sache que des cellules dérivées des cellules hématopoïétiques du donneur peuvent être observées dans le cerveau de patients 48 heures après la greffe, le turn-over des cellules microgliales est un processus relativement lent, même s'il peut perdurer pendant des années, comme le suggère des améliorations observées jusqu'à 5 ans après greffe chez des patients ALD.

La greffe allogénique de moelle osseuse est ainsi la seule thérapeutique ayant démontré son efficacité dans les atteintes cérébrales de l'ALD. Toutes les approches thérapeutiques (huile de Lorenzo, statines) visant à normaliser les taux plasmatiques d'AGTLC ont été jusqu'ici des échecs, peut-être tout simplement parce qu'elles ne permettent pas de normaliser les taux intra-cérébraux d'AGTLC chez les patients (c'est du moins ce que suggère l'application de ces deux traitements aux souris ALD). La greffe allogénique de moelle osseuse reste cependant associée à une lourde mortalité et n'est pas toujours possible faute de donneur. Alors que des résultats à long terme suggèrent que la greffe de moelle osseuse pourrait même être efficace dans les formes adultes d'ALD qui touchent la moelle épinière, il est exclu aujourd'hui de proposer cette approche à des patients adultes atteints d'AMN isolée, vu le risque encore plus important de la greffe à cet âge. Une auto-transplantation de cellules souches hématopoiétiques $\left(\mathrm{CD} 34^{+}\right)$génétiquement corrigées ex vivo pourrait permettre de résoudre la majeure partie des problèmes soulevés par la greffe allogénique. L’utilisation de vecteurs rétro-viraux murins permet de corriger in vitro
20 à $30 \%$ des cellules CD34+ ${ }^{+}$de patients ALD [21]. Ces résultats démontrent la faisabilité de cette approche mais ne permettent pas d'envisager un essai clinique pour le moment: (1) in vivo chez les patients, le pourcentage de correction serait bien moindre et totalement inefficace sur le plan clinique; (2) les cellules hématopoïétiques normales n'ont aucun avantage sélectif sur les cellules ALD mutées, contrairement à ce qui est observé dans certains déficits immunitaires [22]. L'utilisation de vecteurs lentiviraux dérivés de HIV pourrait en revanche permettre d'obtenir une efficacité de correction bien supérieure [23-25].

Même si la mutation du gène ALD n'affecte que secondairement les oligodendrocytes, il faudra certainement explorer aussi la possibilité de greffe intra-cérébrale d'oligodendrocytes pour obtenir une remyélinisation plus complète des lésions. La greffe peut être allogénique, mais il est clair qu'une greffe des propres cellules oligodendrogliales précurseurs du patient (après correction du déficit génétique ex vivo) ou même, pourquoi pas, de cellules souches autologues d'autre origine (que l'on pourrait faire différencier en oligodendrocytes avant réinjection) serait à terme préférable. Les récents progrès réalisés dans l'isolement de précurseurs oligodendrogliaux, la possibilité récemment entrouverte mais non encore démontrée de faire se différencier des cellules souches hématopoḯtiques en neurones ou en cellules gliales, la possibilité de corriger génétiquement ces cellules $e x$ vivo, et même de les expandre avant ré-injection, rend cette approche envisageable à long terme, même s'il reste encore beaucoup de chemin à parcourir. Restera enfin le difficile problème de traiter les formes cérébrales d'ALD à un stade évolué. Sur la base d'une certaine ressemblance des lésions inflammatoires de l'ALD avec celles de la sclérose en plaques, différents traitements ayant montré un certain effet dans cette dernière maladie ( $\beta$-interféron, immunoglobulines, mitoxantrone, etc.) ont été essayés dans l'ALD. Dans tous les cas, cela a été un échec. La raison en est 
vraisemblablement que, malgré certaines similitudes, les mécanismes responsables de l'atteinte cérébrale inflammatoire de l'ALD sont en fait différents de ceux mis en jeu dans la sclérose en plaques [18]. Ceci devrait conduire à une complète révision de nos approches thérapeutiques dans ces formes graves d'ALD qui restent aujourd'hui totalement réfractaires à tout traitement

\section{RÉFÉRENCES}

1. Perry VH, Anderson PB, Gordon S Macrophages and inflammation in the CNS. Trends Neurosci 1993; 167: 268-73.

2. Eglitis MA, Mezey E. Hematopoietic cells differentiate into both microglia and macroglia in the brains of adult mice. Proc Natl Acad Sci USA 1997; 94: 4080-5.

3. Moser HW, Tutschka PJ, Brown FRI, et al. Bone marrow transplant in adrenoleukodystrophy. Neurology 1984; 34 : 1410-7.

4. Aubourg P, Blanche S, Jambaqué I, et al. Reversal of early neurologic and neuroradiologic manifestations of X-linked adrenoleukodystrophy by bone marrow transplantation. N Engl J Med 1990 ; 322 : 1860-6.

5. Shapiro E, Krivit W, Lockman L, et al. Long-term effet of bone marrow transplantation for childhood-onset cerebral X-linked adrenoleukodystrophy. Lancet 2000 ; 356: 713-8.

6. Moser HW. Adrenoleukodystrophy: phenotype, genetics, pathogenesis and therapy. Brain 1997; 120: 1485-508.

7. Aubourg P. In: Vinken PJ, Bruyn G.W, Moser,H.W. eds. Handbook of Clinical Neurology: Neurodystrophies and Neurolipidoses. Amsterdam : Elsevier 1997: 447-83.

8. Dubois-Dalcq M, Feigenbaum V, Aubourg $\mathrm{P}$. The neurobiology of X-linked adrenoleukodystrophy, a demyelinating peroxisomal disorder. Trends Neurosci 1999; 22: 4-12.
9. Kobayashi T, Shinnoh N, Kondo A, Yamada T. Adrenoleukodystrophy proteindeficient mice represent abnormality of very long chain fatty acid metabolism. Biochem Biophys Res Commun 1997; 232: 631-6.

10. Lu JF, Lawler AM, Watkins PA, et al. A mouse model for X-linked adrenoleukodystrophy. Proc Natl Acad Sci USA 1997; 94 : 9366-71.

11. Forss-Petter S, Werner H, Berger J, et al. Targeted inactivation of the X-linked adrenoleukodystrophy gene in mice. I Neurosci Res 1997; 50 : 829-43.

12. Mosser I, Douar AM, Sarde CO, et al. Putative X-linked adrenoleukodystrophy gene shares unexpected homology with ABC transporters. Nature 1993; 361 : 726-30.

13. Liu L-X, Janvier K, Berteaux-Lecellier V, Cartier N, Benarous R, Aubourg P. Homoand heterodimerization of peroxisomal ATP-binding cassette half-transporters. J Biol Chem 1999; 274: 32738-43.

14. Fouquet F, Zhou JM, Ralston E, et al. Expression of the adrenoleukodystrophy protein in the human and mouse central nervous system. Neurobiol Dis 1997; 3: 27185 .

15. Feigenbaum A, Gélot A, Casanova P, Daumas-Duport C, Aubourg P, DuboisDalcq M. Apoptosis in central nervous system of cerebral adrenoleukodystrophy patients. Neurobiol Dis 2000 (sous presse).

16. Ho JK, Moser H, Kishimoto Y, Hamilton JA. Interactions of a very long chain fatty acid with model membranes and serum albumin - Implications for the pathogenesis of adrenoleukodystrophy. J Clin Invest 1995 ; 96: $1455-63$.

17. Min K-T, Benzer S. Preventing neurodegeneration in the drosophilia mutant bubblegum. Science 1999; 284: 1985-8.

18. Aubourg P, Dubois-Dalcq M. X-linked adrenoleukodystrophy enigma: How does the ALD peroxisomal transporter mutation affect CNS glia? Glia 2000; 29 : 186-90.
19. Lannuzel A, Aubourg P, Tardieu M. Excessive production of tumour necrosis factor alpha by peripheral blood mononuclear cells in X-linked adrenoleukodystrophy. Eur J Paed Neurol 1998; 1 : 27-32.

20. Peters C, Shapiro E, Ziegler R, Krivit W. $\mathrm{In}$ : Peters $\mathrm{C}$, ed. Correction of genetic diseases by transplantation $V$. London: The Cogent Trust, 2000 (sous presse).

21. Doerflinger N, Miclea JM, Lopez J, et al. Retroviral transfer and long-term expression of the adrenoleukodystrophy gene in human CD $34^{+}$cells. Hum Gene Ther 1998; 9 : 1025-36.

22. Cavazzana-Calvo M, Hacein-Bey S, de Saint-Basile G, et al. Gene therapy of human severe combined immunodeficiency (SCID)-X1 disease. Science 2000; 288: 66972 .

23. Miyoshi H, Smith KA, Mosier DE, Verma IM, Torbett BE. Transduction of human CD34+ cells that mediate long-term engraftment of NOD/SCID mice by HIV vectors. Science 1999 ; 283 : 682-6.

24. Zennou V, Petit C, Guetard D, Nerhbass U, Montagnier L, Charneau P. HIV-1 genome nuclear import is mediated by a central DNA flap. Cell 2000; 101 : 173-85.

25. May C, Rivella S, Callegari J, et al. Therapeutic haemoglobin synthesis in $\beta$-thalassaemic mice expressing lentivirus-encoded human B-globin. Nature $2000 ; 406$ : 82-5.

\section{Patrick Aubourg}

Inserm U. 342, Hôpital Saint-Vincent-dePaul, 82, avenue Denfert-Rochereau, 75014 Paris, France.

\section{TIRÉS À PART}

P. Aubourg. 